\title{
Assessing early-onset hallucinations in the touch-screen generation
}

Morgane Demeulemeester, Fréderic Kochman, Benjamin Fligans, Ahmed J. Tabet, Pierre Thomas and Renaud Jardri

\section{Summary}

The increasing development of apps for digital devices provides an opportunity for new instruments to assess hallucinations in young individuals. Here we present the Multisensory HAllucinations Scale for Children (MHASC), dedicated to assessing complex early-onset hallucinations. The MHASC will soon be translated into multilanguage versions with the support of the International Consortium of Hallucination Research.

\section{Declaration of interest}

R.J., B.F., A.J.T., M.D. are the developers of the MHASC app. MHASC is a trademark of Université de Lille \& Centre Hospitalier Régional Universitaire de Lille.
Morgane Demeulemeester (pictured) is a PhD student at Université de Lille and a psychologist at the Lautréamont Clinic. Fréderic Kochman is a psychiatrist at the Lautréamont Clinic. Benjamin Fligans is a designer at Université de Lille. Ahmed J. Tabet is a computer engineer at Université de Lille. Pierre Thomas is a professor of psychiatry at Université de Lille and Centre Hospitalier Régional Universitaire de Lille. Renaud Jardri is a professor of child \& adolescent psychiatry at Université de Lille and Centre Hospitalier Régional Universitaire de Lille and principal investigator of the MHASC project.

\section{The rise of mobile health (mHealth) apps}

Increased access to tablets and smartphones means that children born during and after the 2000s are the so-called 'touch-screen generation'. Digital devices leave few areas untouched and children appear at the forefront of this technological revolution (for example in the USA, one in four teenagers owned a tablet in $2013,{ }^{1}$ and this proportion might reach $60 \%$ by the end of 2017). Being diagnosed with a mental disorder does not affect the use of digital devices (for example a recent survey indicated that up to $60 \%$ of individuals with a schizophrenia spectrum disorder already own and use mobile devices ${ }^{2}$ ), which paves the way for specific mHealth apps. More than $10^{4} \mathrm{mHealth}$ apps are available for download from popular online stores, however, insufficient effort has been devoted to the design and psychometric validation of these apps, which warrants caution on the part of both consumers and clinicians with respect to the security of medical data-exchange. ${ }^{3}$

A first strategy in developing mHealth apps has been the digitalisation of pre-existing psychometric tools to improve participants' engagement and follow-up or to reduce diagnosis times by using automatic electronic scoring schemes. In child and adolescent psychiatry, computerised versions of the DICA-R (Diagnostic Interview for Children and Adolescents - revised), the Dominic Interactive or the MMPI-2-RF (Minnesota Multiphasic Personality Inventory - restructured form) have, for example, been made available. For the majority of these tools, combined auditory and visual assessment strategies are presented to generate a better understanding, rather than using more traditional questionnaires as well as decreased dependency on the child's vocabulary or literacy. In these preliminary experiences, children enjoyed the computer and completed the assessment with little help.
A second strategy has been to design tools able to offer new avenues of psychopathological assessment, such as by collecting data on time-sensitive symptoms using ecological momentary assessment (EMA) paradigms. Modern EMA effectively takes advantage of mobile devices and has been shown to be useful in the development of individually tailored psychoeducation ${ }^{4}$ and to improve medication adherence in schizophrenia. ${ }^{5}$

\section{Assessing hallucinations in young people}

In this general context, how does media technology change the way we assess hallucinations in children and adolescents? Hallucinations are intimate and occasionally scary experiences; children rarely mention these experiences spontaneously to their therapists and parents. Early-onset hallucinations that present in high rates ( $8 \%$ of the general population) during childhood are typically described as more vivid and particularly multisensory compared with adult experiences. ${ }^{6}$ Because their characteristics and severity may vary, obtaining deeper clinical insight into early-onset hallucinations is crucial.

Several shortcomings in existing methods for assessing hallucinations can be identified. A first limitation is that although the current 'paper-and-pencil' tools available for assessing earlyonset hallucinations have been adapted for different age groups these questionnaires primarily focus on voice hearing in children and therefore do not assess the sensory complexity of early-onset hallucinations (i.e. extra-auditory experiences). A second limitation is that investigator-based interviews, often considered the gold standard for assessing early-onset hallucinations, are time-consuming and may affect children's replies, especially when they want to reveal some embarrassing information to the interviewer. The validity of using self-report measures rather than interviews in participants with potentially poorer insight has raised some concerns but they have, nevertheless, been shown to be effective, particularly for hallucinatory experiences. ${ }^{7}$ Digital formats may notably help to overcome these issues and allow the advantages of both approaches to be combined. The concept of designing and validating a new category of mHealth app, named the Multisensory HAllucinations Scale for Children (MHASC), dedicated to the assessment of early-onset hallucinations emerged in this context.

MHASC is an intermediate tool between self- and heteroassessment. By fully exploiting the advantages of $17-25 \mathrm{~cm}$ 
(7-10 inch) tablets (because of animations, avatars and text-size, smartphones do not permit optimal use of the MHASC), this app is intended to elicit the direct participation of children and adolescents in a user-friendly way that is capable of increasing the reliability of descriptions of early-onset hallucinations while avoiding overloading with distracting stimulation. As a medical device, two different interfaces have been implemented: (a) a 'professional dashboard' for the adult that initiates the assessment and allows access to the analysis tools; (b) a 'child/adolescent interface' (7- to 18-years-olds) (Fig. 1). This second interface begins with the customisation of the child's avatar, which will accompany her or him during the entire session, providing help when necessary. In this regard, the MHASC can be viewed as a game and at the same time a highly standardised test (also known as 'serious games') that will enable children to freely communicate their own perspectives with regard to early-onset hallucinations. This intermediate status of MHASC (between self- and heteroassessment) removes the above-mentioned barriers and tends to permit the evaluator's presence to fade away, a point judged crucial for two reasons. First, the presence of others has been shown to directly affect the validity of self-reports in youth. ${ }^{8}$ Second, devices allow genuine mobility, whereby children are no longer confined to the restrictions of the clinical ward or the therapist's office, allowing for a new conception of longitudinal assessment of early-onset hallucinations.
To complement the quantification of common features of hallucinatory experiences that will be combined to generate severity scores and hallucinatory profiles in various sensory modalities (such as frequency, intensity, conviction, insight, degree of control, discomfort in daily life, distress, emotional valence, coping strategy), MHASC will assess the development and acquisition of cognitive functions that may help the clinician to diagnose or hypothesise, such as theory of mind or mental imagery (ToM and Mentor modules respectively, see Fig. 1). This complementary assessment aims to distinguish early-onset hallucinations from immature responses to imaginary companions that, by several definitions, may fit the criteria for hallucinations. A positive correlation, for example, has been observed between the presence of a parentally corroborated imaginary companion and the tendency to hear words when the child was placed in a noisy or ambiguous test environment. ${ }^{9}$ However, the presence of imaginary companions has been associated with positive developmental outcomes, such as theory of mind performance, ${ }^{6}$ which indicates that imaginary companions should certainly not be considered markers of psychosis.

The cognitive modules of the MHASC have been independently validated. A first version of the MHASC is currently being validated in France and will progressively integrate new modules during 2015 (such as EMA). Given that we are

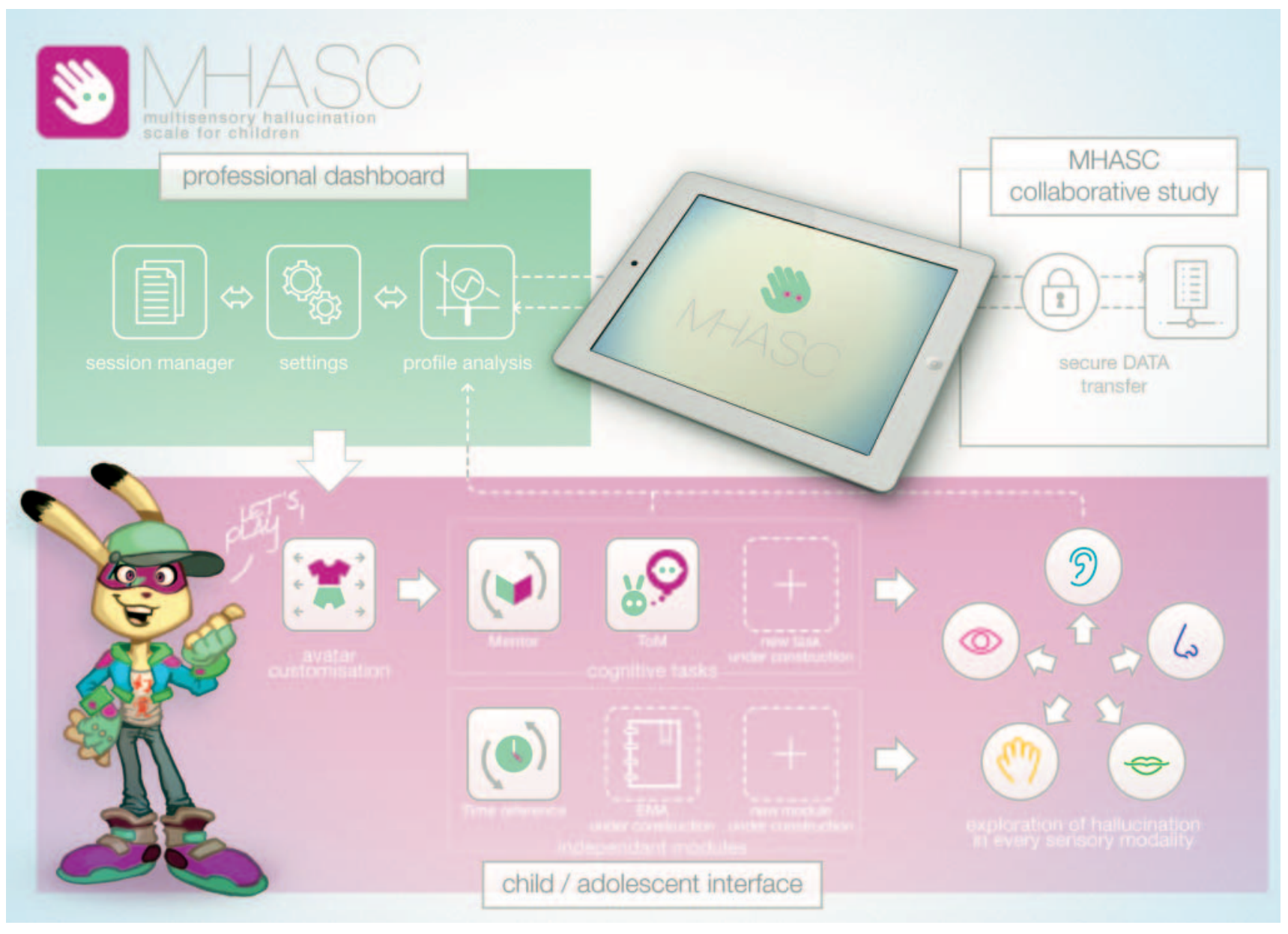

Fig. 1 The modular architecture of the MHASC ${ }^{\odot}$ app.

Two levels of navigation are provided: one dedicated to professionals (upper panel) and the other dedicated to children and adolescents (lower panel). Future developments will allow the addition of new modules to these two dashboards (for example an ecological momentary assessment module (EMA)...). 
convinced that the combination of such technological advancements may lead to significant benefits in the rigorous assessment of early-onset hallucinations, MHASC appears to be an ideal mHealth tool for multicenter research projects. In this way, the MHASC will be able to take advantage of the increasing amount of research conducted on data-gathering through apps. ${ }^{10}$ Two storage options will, for example, be proposed for the collected data: (a) systematic saving on the device onboard flash memory; and in complement (b) the professional will have the option to authorise automated transmission of an anonymous summary to a secured server and access to multicentre analysis tools (see Fig. 1). The final French version is expected in 2015 and MHASC will also be translated into multilanguage versions (English and Dutch are scheduled by the end of 2015) with the support of the International Consortium of Hallucination Research, a platform that was originally established to motivate research in hallucinations, to promote scientific rigor and to enhance international collaborative work on the topic. ${ }^{11}$

Morgane Demeulemeester, Université de Lille, SCA-Lab, PSYChiC team, Lille and Lautréamont Clinic, ORPEA-CLINEA Group, LOOS; Fréderic Kochman, MD, Lautréamont Clinic, ORPEA-CLINEA Group, Loos; Benjamin Fligans, Université de Lille, SCA-Lab, PSYChiC team, Lille; Ahmed J, Tabet Université de Lille, PolyTech, Lille; Pierre Thomas, MD, PhD, Université de Lille, SCA-Lab, PSYChiC team, and Centre Hospitalier Régional Universitaire (CHRU) de Lille, Adult Psychiatry Department, Lille; Renaud Jardri, MD, PhD, Université de Lille, SCA-Lab, PSYChic team, and Centre Hospitalier Régional Universitaire (CHRU) de Lille, Child \& Adolescent Psychiatry Department, Lille, France

Correspondence: Renaud Jardri, MD, PhD, Service de Psychiatrie de l'enfant et de I'adolescent, Hôpital Fontan, CHRU de Lille, CS 70001, 59037 Lille cedex, France. Email: renaud.jardri@chru-lille.fr

First received 26 Dec 2013, final revision 5 Jul 2014, accepted 7 Oct 2014

\section{Funding}

R.J. received funding from the Deniker Foundation for the MHASC project. M.D. held a doctoral fellowship from the Association National de la Recherche et de la Technologie (ANRT)

\section{References}

1 Madden M, Lenhart A, Duggan M, Cortesi S, Gasser U. Teens and Technology 2013. Pew Research 13 March 2013, (http://www.pewinternet.org/2013/03/ 13/teens-and-technology-2013/)

2 Ben-Zeev D, Kaiser SM, Brenner CJ, Begale M, Duffecy J, Mohr DC Development and usability testing of FOCUS: a smartphone system for self-management of schizophrenia. Psychiatr Rehab J 2013; 36: 289-96.

3 Powell $A C$, Landman $A B$, Bates DW. In search of a few good apps. JAMA 2014; 311: 1851-2.

4 Oorschot M, Lataster T, Thewissen V, Wichers M, Myin-Germeys I. Mobile assessment in schizophrenia: a data-driven momentary approach. Schizophr Bull 2012; 38: 405-13.

5 Granholm E, Ben-Zeev D, Link PC, Bradshaw KR, Holden JL. Mobile Assessment and Treatment for Schizophrenia (MATS): a pilot trial of an interactive text-messaging intervention for medication adherence, socialization, and auditory hallucinations. Schizophr Bull 2012; 38: 414-25.

6 Jardri R, Bartels-Velthuis AA, Debbané M, Jenner JA, Kelleher I, Dauvilliers Y, et al. From phenomenology to neurophysiological understanding of hallucinations in children and adolescents. Schizophr Bull 2014; 40 (suppl 4): s221-32.

7 Hamera EK, Schneider JK, Potocky M, Casebeer MA. Validity of selfadministered symptom scales in clients with schizophrenia and schizoaffective disorders. Schizophr Res 1996; 19: 213-9.

8 Vereecken CA, Maes L. Comparison of a computer-administered and paper-and-pencil-administered questionnaire on health and lifestyle behaviors. J Adolesc Health 2006; 38: 426-32.

9 Fernyhough C, Bland K, Meins E, Coltheart M. Imaginary companions and young children's responses to ambiguous auditory stimuli: implications for typical and atypical development. J Child Psychol Psychiatry 2007; 48: 1094-101.

10 Killingsworth MA, Gilbert DT. A wandering mind is an unhappy mind. Science 2010; 30: 932.

11 Waters F. Multidisciplinary approaches to understanding auditory hallucinations in schizophrenia and nonschizophrenia populations: the International Consortium on Hallucination Research. Schizophr Bull 2012; 38: 693-4. 